\title{
Massive cerebral venous thrombosis and venous watershed infarction as late complications of COVID-19: a case report
}

\author{
Fatma Şimşek ${ }^{1}$ (D) Ravza Tosunoğlu ${ }^{1}$ (i)
}

Received: 25 April 2021 / Accepted: 18 July 2021 / Published online: 10 August 2021

(c) Fondazione Società Italiana di Neurologia 2021

\section{Dear Editor-in-Chief,}

In this case report, a male patient who was recovered from COVID-19 with mild symptoms but has developed massive CVT accompanied by venous watershed infarcts as late complications of COVID-19 is presented.

The 39-year-old male patient subject to this case report presented to the emergency service with severe headache that started 2 days ago and persisted since then. Vital signs were stable. Neurological examination was normal except for bilateral papilledema. Three months before his hospitalization due to severe headache, he had tested positive for COVID-19, based on the real-time reverse transcriptase polymerase chain reaction (RT-PCR) test performed via nasopharyngeal swab. Neither he nor his family had a history of venous thromboembolism. The computed tomography (CT) scan of his brain taken at the emergency service revealed an increase in calibration and density in bilateral transverse sinus, straight sinus, and superior sagittal sinus (Fig. 1). The results of the laboratory tests of the patient, who was considered to have CVT based on the brain CT scan, are given in Table 1 . The high resolution thorax computed tomography of the patient revealed minimal imperceptible ground glass

\section{Highlights}

- Cerebral venous thrombosis (CVT) is a rare complication of COVID-19 infection.

- Late neurological complications can be seen in COVID-19 infection.

- Venous watershed infarcts are rare, and CVT is also rare.

- CVT is more common in cases with a mild course of COVID infection.

Fatma Şimşek

klamaks@hotmail.com

Ravza Tosunoğlu

Ravza.tosunoglu@gmail.com

1 Department of Neurology, Faculty of Medicine, Ataturk University, Erzurum, Turkey images in the bilateral lower lobes. This result was attributed to COVID-19. The patient was hospitalized, and he was started on $2 \times 60 \mathrm{mg} /$ day subcutaneous low molecular weight heparin, $2 \times 1 \mathrm{~g} /$ day intravenous ceftriaxone, $4 \times 125 \mathrm{cc}$ intravenous mannitol, and $3 \times 250 \mathrm{mg}$ oral acetazolamide. Mannitol was given for 3 days, ceftriaxone for 2 weeks, and low molecular weight heparin for 3 weeks. Acetazolamide was continued until the headache was relieved and the papilledema subsided. RT-PCR test was performed again from the nasopharyngeal swab on admission, and it was negative. His immunoglobulin $\mathrm{G}(\mathrm{IgG})$ antibody level was found to be $3.34 \mathrm{~g} / \mathrm{L}$. He tested negative for prothrombin gene mutation test, which was administered in search for the etiology. He was found to have heterozygous methylene tetrahydrofolate reductase and factor $\mathrm{V}$ Leiden gene mutations. The results of his anti-nuclear antibody, anti-ds-DNA, antiphospholipid antibody, and anticardiolipin antibody tests came out as negative. Cranial magnetic resonance imaging (MRI) revealed venous infarct area in the right thalamus; MRI and magnetic resonance venography (MRV) revealed bilateral transverse sinus, straight sinus, superior sagittal sinus, right internal cerebral vein, and thrombus in the right thalamostriate vein; and diffusion-weighted images revealed watershed infarct areas restricting diffusion in the border regions of the deep white matter (Fig. 2). Carotid-vertebral artery Doppler ultrasonography did not reveal anything abnormal, as did electrocardiography, echocardiography, Holter monitor test, and magnetic resonance angiography. After 3 weeks of treatment, the patient's headaches almost halved, and his C-reactive protein (CRP) levels returned to the normal. He was started on warfarin sodium, having set the related international correction rate (INR) to be in the range of 2-2.5, and was then discharged, having scheduled a follow-up visit for 1 month after the date of discharge. He was still being followed up until the time he presented to the emergency service with severe headache.

It was reported in numerous studies that COVID-19 causes neurological symptoms. Neurological findings were 


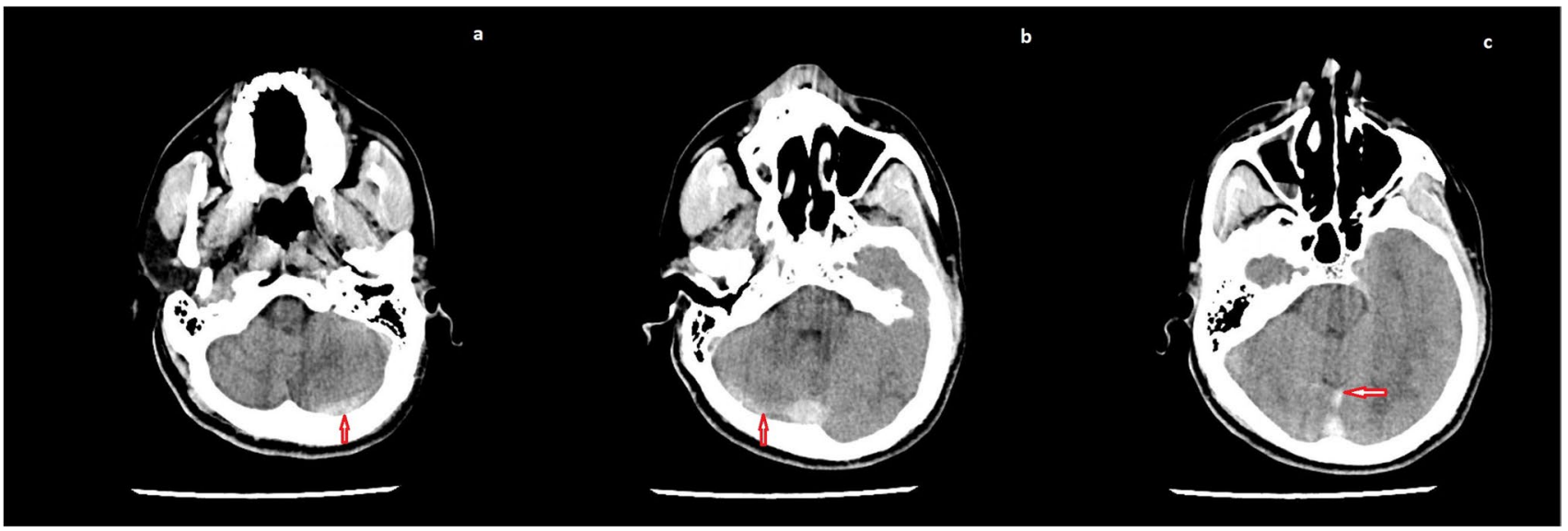

Fig. 1 CT scan of the brain revealed increases in densities, which are consistent with hyperdense cerebral venous thrombosis, in the a right transverse sinus, $\mathbf{b}$ straight sinus, and $\mathbf{c}$ left transverse sinus

Table 1 Laboratory data of the patient

\begin{tabular}{cl}
\hline Sample type and normal range & Patient data \\
\hline WBC $\left(3.9-10.8 \times 10^{3} / \mu \mathrm{L}\right)$ & $\mathbf{1 2 . 7 1 \times 1 0 ^ { 3 }}$ \\
Neutrophils $\left(1.91-7.61 \times 10^{3} / \mu \mathrm{L}\right)$ & $\mathbf{9 . 7 8} \times 10^{3}$ \\
Lymphocytes $\left(1.16-3.61 \times 10^{3} / \mu \mathrm{L}\right)$ & $1.38 \times 10^{3}$ \\
Hemoglobin $(14.8-18.3 \mathrm{~g} / \mathrm{dL})$ & 15.2 \\
Platelets $\left(145-345 \times 10^{3} / \mu \mathrm{L}\right)$ & $184 \times 10^{3}$ \\
PT $(12-16 \mathrm{sn})$ & 14.6 \\
APTT $(25-35 \mathrm{~s})$ & 32.4 \\
INR $(0.9-1.3)$ & 1.08 \\
Fibrinogen $(245-400 \mathrm{mg} / \mathrm{dL})$ & $\mathbf{5 2 6}$ \\
CRP $(0-5 \mathrm{mg} / \mathrm{L})$ & $\mathbf{5 3 . 3}$ \\
ESR $(0-20 \mathrm{~mm} / \mathrm{h})$ & $\mathbf{2 2}$ \\
AT3 $(80-120)$ & 81 \\
Protein C $(70-130 \%)$ & 91 \\
Protein S $(65-140 \%)$ & 92 \\
Homocysteine $(5-15 \mathrm{umol} / \mathrm{L})$ & 13.3 \\
COVID-19 IGG $(0-1)$ & $\mathbf{3 . 3 4}$ \\
\hline
\end{tabular}

$W B C$ white blood cell, $P T$ prothrombin time, $A P T T$ activated partial thromboplastin time, CRP C reactive protein, ESR erythrocyte sedimentation rate, AT3 antithrombin 3, INR international correction rate

observed in $36.4 \%$ of the cases, and it was found that these neurological findings were associated with stroke and coagulopathy in patients with severe COVID-19 infection [1]. A systematic review revealed that the patients who have developed CVT due to COVID-19 are generally young patients with mild symptoms and without any comorbidities [2]. Patients (33.3\%) who developed CVT due to COVID-19 infection were determined to have Internal cerebral veins and straight sinus involvement, and morbidity and mortality rates were reported to be higher in patients who had thrombosis in these regions [3]. In the literature, higher mortality rates were reported in patients with bilateral involvement in deep cerebral veins. In parallel, the fact that the patient presented in this case report had only unilateral deep venous involvement contributed to the good prognosis. However, contrary to the cases reported in the literature, in whom CVT development was reported to have been observed in the first few weeks following the COVID-19 infection, the fact that clinical findings of CVT emerged in the patient presented herein 3 months after the COVID-19 infection suggests that COVID-19-related complications may occur up to 2-3 months after the disease.

Acute infarction areas in the deep cerebral white matter observed in the patient presented herein were generally observed in the hypotensive cerebral infarction. There are case reports in the literature with similar findings, in which venous watershed infarction areas rarely seen after CVT were reported $[4,5]$. As was observed in the case presented herein, acute infarct areas can be observed due to impaired drainage in thrombosis emerged in the deep veins in this region. One of the possible factors contributing to late intracranial venous thrombosis in a patient with a factor $\mathrm{V}$ Leiden heterozygous mutation is a previous COVID infection. In conclusion, this case report is presented to make a contribution to the literature in that it features CVT development as a late complication of COVID-19 infection, and that it is the first case in which watershed infarct areas accompanying CVT were observed after COVID-19. 


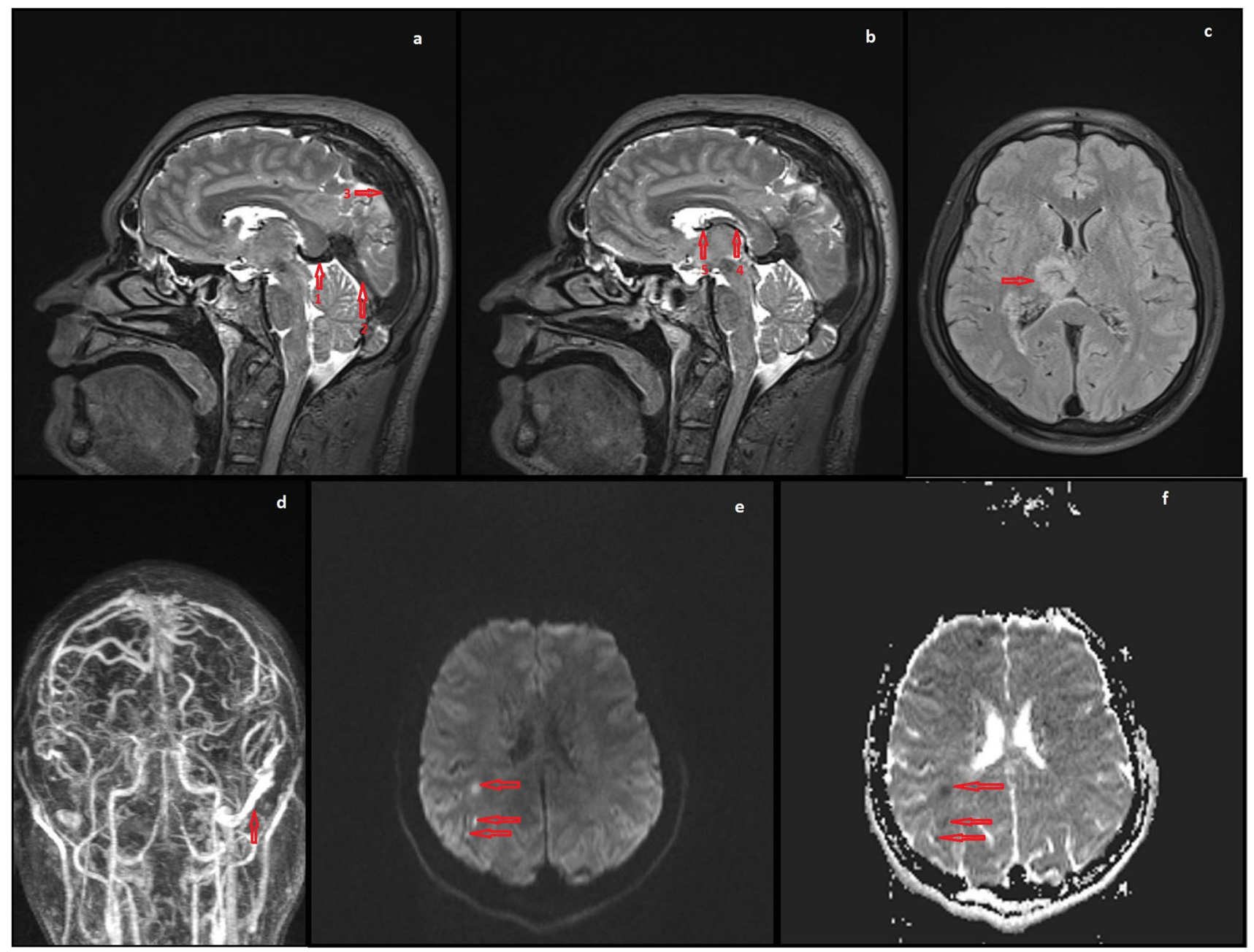

Fig. 2 MRI revealed 1, Galen vein; 2, straight sinus; 3, superior sagittal sinus (a); 4, right internal cerebral vein; and 5 right thalamostriate vein (b) filling defects compatible with hypointense thrombus in the sagittal T2 sequence. Axial FLAIR (fluid attenuated inversion recovery) sequence revealed hyperintense area (c) compatible with

\section{Declarations}

Conflict of interest The authors declare no competing interests.

\section{References}

1. Zhang Y, Xiao M, Zhang S, Xia P, Cao W, Jiang W et al (2020) Coagulopathy and antiphospholipid antibodies in patients with Covid-19. N Engl J Med 382:e38

2. Tu TM, Goh C, Tan YK, Leow AS, Pang YZ, Chien J et al (2020) Cerebral venous thrombosis in patients with COVID-19 infection: a case series and systematic review. J Stroke Cerebrovasc Dis 29(12):105379. https://doi.org/10.1016/j.jstrokecerebrovasdis. 2020.105379 venous infarction in the right thalamus. MRV revealed flow in the left sigmoid sinus but did not reveal the other superficial veins (d). Diffusion-weighted images revealed hyperintense in B1000 in the right parietooccipital region (e), hypointense in ADC (f), and venous watershed infarct areas in which diffusion restriction was observed

3. Yeo L, Lye P, Yee K, Cunli Y, Ming T, Ho A et al (2020) Deep cerebral venous thrombosis treatment: endovascular case using aspiration and review of the various treatment modalities. Clin Neuroradiol 30:661-670

4. Singh R-J, Saini J, Holla VV, Kamble N (2017) Venous infarcts mimicking large vessel arterial disease: watershed lesions in deep cerebral venous thrombosis. J Stroke Cerebrovasc Dis 2:455-456

5. Washida K, Kowa H, Tsuji Y, Sekiguchi K, Kanda F, Toda T (2016) Multiple deep white matter hyperintense lesions on diffusion-weighted imaging: early sign of straight sinus thrombosis. J Stroke Cerebrovasc Dis 25:e131-e133

Publisher's note Springer Nature remains neutral with regard to jurisdictional claims in published maps and institutional affiliations. 